\title{
Protocolo para el manejo de placenta percreta con cesárea, embolización uterina e histerectomía diferida
}

\author{
Protocol for the management of percrete placenta with cesarean, uterine \\ embolization, and deferred hysterectomy
}

Cesar Eduardo Jiménez-Jiménez¹, Jorge Ernesto Niño-González², Angy Lorena Meneses-Parra³

1 Médico, especialista en Cirugía general y Cirugía vascular; jefe, Servicio de cirugía Vascular y Endovascular, Hospital Universitario Clínica San Rafael, Bogotá, D.C., Colombia. Correo: cesarejmd@yahoo.com

2 Médico, especialista en Ginecología y Obstetricia; jefe, Unidad de Obstetricia y Ginecología, Hospital Universitario Clínica San Rafael, Bogotá, D.C., Colombia.

3 Médica, residente de Ginecología y Obstetricia, Universidad Militar Nueva Granada, Bogotá, D.C., Colombia.

\section{Resumen}

Introducción. El objetivo de este artículo fue dar a conocer el protocolo institucional del manejo de la placenta percreta como un procedimiento varias horas después de la cesárea, con embolización de arterias placentarias de forma selectivas, previo a la práctica de la histerectomía, y presentar los resultados.

Métodos. Estudio de serie de casos, donde se evaluaron las pacientes con placenta percreta, manejadas durante un año en un hospital de cuarto nivel de complejidad en la ciudad de Bogotá, D.C., Colombia. Se efectuó cesárea fúndica y se dejó la placenta in situ, 48 a 72 horas después se realizó embolización ultra selectiva y luego de 2 a 3 días se procedió a practicar la histerectomía vía abdominal.

Resultados. Se evaluaron 5 pacientes, con paridad de 3,8 embarazos promedio, con diagnóstico de placenta percreta. El tiempo promedio de espera entre la embolización y la histerectomía fue de 1,6 días. No se presentaron complicaciones asociadas a la embolización, ni morbimortalidad materno fetal. Los volúmenes de sangrado en promedio durante la histerectomía de cada paciente fueron de $1160 \mathrm{ml}$.

Conclusión. Existen datos limitados sobre el tratamiento óptimo del acretismo placentario. La sospecha diagnóstica permite planificar de forma favorable el manejo intraparto y, es por ello, que el surgimiento de nuevas técnicas, como la embolización de arterias placentarias, constituyen alternativas para un manejo más seguro de las pacientes.

Palabras claves: placenta accreta; placenta previa; retención de la placenta; cesárea; histerectomía; embolización terapéutica.

Fecha de recibido: 28/06/2021 - Fecha de aceptación: 15/09/2021 - Publicación en línea: 07/10/2021

Correspondencia: Cesar Eduardo Jiménez, Carrera 8 \# 17-45 Sur, Hospital Universitario Clínica San Rafael, Bogotá, D.C., Colombia, 110411. Teléfono: (+57)3142976816. Correo: cesarejmd@yahoo.com

Citar como: Jiménez-Jiménez CE, Niño-González JE, Meneses-Parra AL. Protocolo para el manejo de placenta percreta con cesárea, embolización uterina e histerectomía diferida. Rev Colomb Cir. 2022;37:106-14. https://doi.org/10.30944/20117582.945

Este es un artículo de acceso abierto bajo una Licencia Creative Commons - BY-NC-ND https://creativecommons.org/licenses/by-nc-nd/4.0/deed.es 


\begin{abstract}
Introduction. The objective of this article was to present the institutional protocol for the management of percrete placenta as a procedure several hours after cesarean section, with selective embolization of placental arteries, prior to the practice of hysterectomy, and to present the results.

Methods. Case series study, where patients with percrete placenta were evaluated, managed for 1 year in a hospital of fourth level of complexity in the city of Bogotá, Colombia. A fundic cesarean section was performed and the placenta was left in situ, 48 to 72 hours later an ultra-selective embolization was performed, followed by an abdominal hysterectomy after 2 to 3 days.

Results. Five patients with a diagnosis of placenta percreta were evaluated; mean wait time between embolization and hysterectomy was 1.6 days. There were no complications associated with embolization, or maternal-fetal morbidity and mortality. Average bleeding volumes during hysterectomy for each patient were $1160 \mathrm{ml}$.

Conclusion. There are limited data on the optimal treatment of percrete placenta. Diagnostic suspicion allows for a favorable planning of intrapartum management and, for this reason, the emergence of new techniques, such as placental artery embolization, constitute alternatives for a safer management of patients.
\end{abstract}

Keywords: percrete placenta; placenta previa; retained placenta; cesarean section; hysterectomy; therapeutic embolization.

\section{Introducción}

El acretismo placentario se entiende como un proceso de inserción anormal de la placenta, secundario a un antecedente quirúrgico en el útero, que con lleva a un error de decidualización en el área cicatricial y permite la profundización anormal del trofoblasto, con sangrado masivo, que ponen en riesgo la vida de la madre y el producto de la gestación ${ }^{1,2}$.

La incidencia de acretismo placentario es de 14,4 por cada 10.000 partos, siendo mayor en mujeres con placenta previa. Esta incidencia ha aumentado de manera paralela con el incremento de las cesáreas, de 1 en 30.000 partos en 1960 y a 1 en 533 partos en 2020, lo que corresponde a un incremento de 60 veces en cinco décadas ${ }^{3}$.

La placenta percreta es la presentación más compleja y peligrosa de las tres formas de acretismo placentario: increta, acreta o percreta. Se ha asociado a sangrado masivo al momento de la cesárea o histerectomía, requiriendo múltiples transfusiones y estancia prolongada en cuidados intensivos, con complicaciones severas dadas por lesiones ureterales o viscerales, además de mortalidad perinatal ${ }^{1}$.
La literatura tiene datos limitados del tratamiento ideal de esta enfermedad, por lo que debemos tener un enfoque organizado, con el fin de disminuir el sangrado y las complicaciones asociadas. El objetivo de este artículo fue dar a conocer el protocolo institucional del manejo de la placenta percreta en el Hospital Universitario Clínica San Rafael, Bogotá, D.C., Colombia, como un procedimiento varias horas después de la cesárea, con embolización de arterias placentarias de forma selectiva, previo a la práctica de la histerectomía, y presentar los resultados en las pacientes atendidas.

\section{Métodos}

\section{Descripción del protocolo}

Posterior al diagnóstico de placenta percreta, se realizó junta medico quirúrgica entre ginecología y cirugía vascular, individualizando a cada paciente según los hallazgos en la imagen ultrasonográfica.

Se explico a la paciente y su familia el procedimiento, sus riesgos y complicaciones, y luego de ello se programó la cesárea, la cual se realizó por vía fúndica mediante laparotomía mediana (figura 1), se extrajo el recién nacido y se proce- 


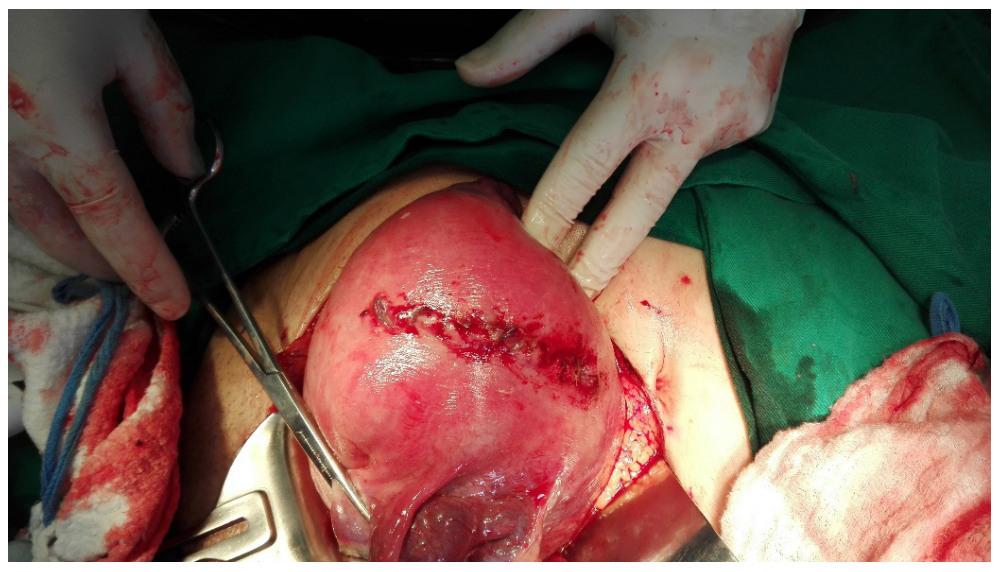

Figura 1. Aspecto de la cirugía donde se observa la cesárea fúndica

dió a la histerorrafia dejando la placenta in situ. A continuación, se practicó control de hemograma diario, pruebas de coagulación y observación clínica estricta, que se registró sin complicaciones.

A las 48 a 72 horas se programó para embolización placentaria ultra-selectiva, por parte del mismo cirujano vascular, ocluyendo de manera selectiva y bilateral todas las arterias anómalas dependientes de la arteria hipogástrica. Entre 48 a 72 horas después se hizo la histerectomía abdominal total, por la misma incisión utilizada para la cesárea, con anestesia general. El procedimiento quirúrgico de cesárea e histerectomía fue realizado por el mismo ginecólogo. Dependiendo del volumen de sangrado intraoperatorio y estado hemodinámico de la paciente se trasladó a habitación convencional o unidad de cuidado intermedio. Durante este periodo se administró antibiótico de amplio espectro (piperacilina/ tazobactam) y anticoagulación profiláctica.

\section{Descripción de la embolización}

El objetivo de la embolización es generar trombosis en el espacio intervelloso, disminuir el riesgo de sangrado y aumentar el proceso de resorción placentaria. Los procedimientos se realizaron en sala de hemodinamia, a través de la arteria femoral común (punción única), bajo anestesia local, con anticoagulación plena con 5000 unidades de Heparina y reversión al final con sulfato de protamina.
A través de un introductor 5 french (Cordis Johnson \& Johnson medical) se accedió a la aorta abdominal con una guía hidrofílica Aquatrak (Cordis-Johnson \& Johnson medical), de 0,035 x 260 centímetros. Se realizó primero un aortograma abdominal con un catéter pigtail (Cordis - Johnson \& Johnson medical) para evaluar la aorta abdominal, arterias iliacas comunes externas e hipogástricas, con el objetivo de determinar las ramas placentarias anómalas.

Posteriormente se canalizaron las arterias hipogástricas y de manera ultra-selectiva las ramas viscerales placentarias con un catéter cobra o Simmons 1 (Cordis - Johnson \& Johnson medical). Se realizan varias inyecciones en DSA (Digital Substraction Angiography) y con fase venosa tardía, y luego de escoger las arterias objetivos, se procedió a canalización con microguía y microcatéter Direction (Boston Scientific, Massachusetts). En primera instancia se realizó embolización con micropartículas (Contour, Boston Scientific, Massachusetts) y microesferas (Embosphere, Merrit Medical Systems Inc.) de 500 a 700 micras, con el objetivo de ir a los vasos más distales y posteriormente se complementaba con microcoils de platino Interlock, de 4 a $6 \mathrm{~mm}$ de diámetro por 40 $\mathrm{cm}$ de longitud (Boston Scientific, Massachusetts). Se efectúo compresión manual después del retiro del introductor y vendaje, con reposo en cama por 12 horas (figura 2). 


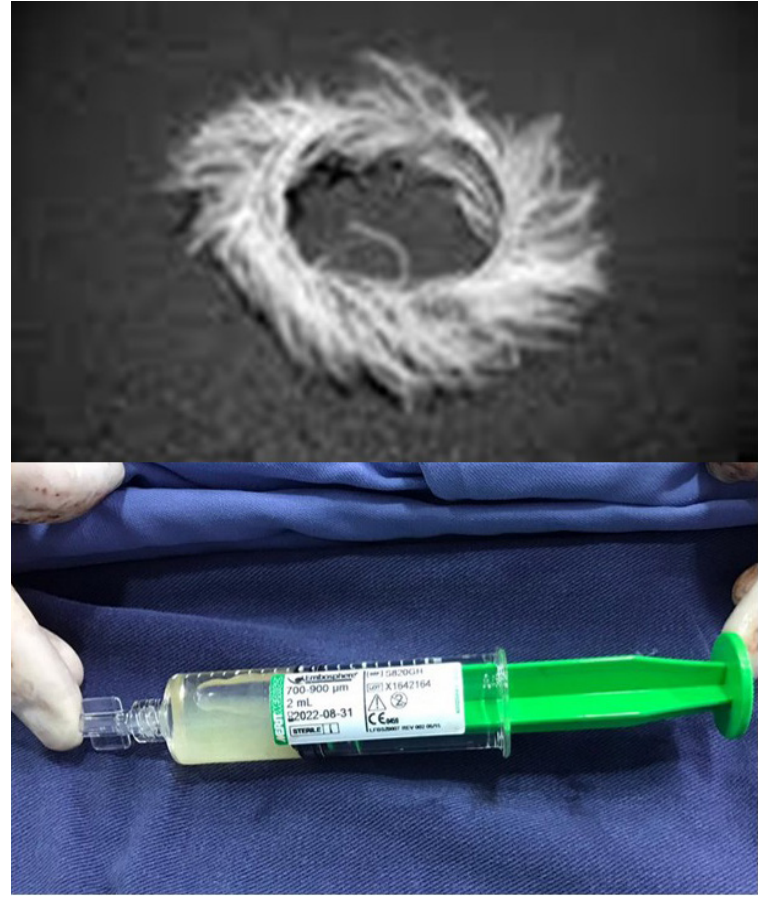

Figura 2. Elementos de embolización con micropartículas y microcoils

\section{Resultados}

En el Hospital Universitario Clínica San Rafael, Bogotá, D.C., Colombia, se evaluaron cinco pacientes con placenta percreta diagnosticada por ecografía en control prenatal, que se llevaron a cesárea e histerectomía programada según protocolo institucional.

Las pacientes tenían una edad promedio de 34,5 años (rango 26 - 41 años) y paridad promedio de 3,8 embarazos (rango 2 - 7 embarazos). Los factores de riesgo identificados para placenta percreta, fueron cesárea previa en 2 y placenta previa en 3 pacientes. No se utilizó metotrexato en ninguno de los casos, y se canalizó solo en un caso los uréteres de manera prequirúrgica.

El promedio de sangrado intraoperatorio fue de $1160 \mathrm{ml}$ (rango 600 - $1500 \mathrm{ml}$ ). El promedio de unidades de glóbulos rojos transfundidas durante la histerectomía fue de 2 unidades (rango 0 - 4). Tres pacientes requirieron traslado a la unidad de cuidados intensivos y 2 se trasladaron a habitación convencional. El promedio de espera entre la cesárea y la embolización fue de 4,8 días (mínimo 3 días - máximo de 8 días) y el promedio de espera entre la embolización y la histerectomía fue de 2,6 días (mínimo 2 días - máximo 5 días)

No existió mortalidad materno fetal ni perinatal a 30 días. No se presentaron complicaciones (lesiones ureterales, viscerales ni sangrado masivo) durante la cesárea o la histerectomía, tampoco se presentaron complicaciones (sangrado, coagulación intravascular diseminada, sepsis, eventos tromboembólicos venosos) durante los periodos de espera entre cesárea y embolización o embolización e histerectomía; no se generaron complicaciones por la embolización (complicaciones del acceso vascular, procedimiento no exitoso, embolización a otros órganos, síndrome postembolización).

\section{Discusión}

El acretismo placentario se debe a ausencia o deficiencia de la capa de Nitabuch, que es una capa de fibrina que se ubica entre la decidua basal y el corion frondoso, debido a una falla en la reconstitución del endometrio/decidua basal, especialmente después de la reparación de un procedimiento quirúrgico, como es la cesárea. En la medida que las cesáreas sigan aumentando a nivel mundial, la frecuencia de esta patología será mayor ${ }^{4}$.

El sangrado genital durante el embarazo es la principal causa de mortalidad en las mujeres gestantes, elevando la morbimortalidad materna (59 \%) y la mortalidad perinatal (9\%) ${ }^{5}$. Puede presentar varias complicaciones que incluyen la necesidad de transfusión sanguínea masiva, infecciones, ruptura uterina y lesiones de vías urinarias y digestivas ${ }^{6}$.

El principal factor de riesgo es el antecedente quirúrgico en el útero (cesárea, miomectomía, legrados uterinos, metroplastias, embolización uterina para miomatosis), pero también se ha asociado con edad avanzada, multiparidad o antecedente de placenta previa ${ }^{7}$.

El acretismo placentario se clasifica según la invasión trofoblástica, como: placenta acreta, la cual se adhiere a miometrio (75 \% de los casos), 
placenta increta, la cual penetra miometrio (15\% de los casos) y placenta percreta, que es aquella que traspasa el miometrio hacia la serosa u órganos adyacentes (5\% de los casos) ${ }^{8}$. Este trabajo incluyó únicamente las pacientes con placenta percreta, debido a su mayor riesgo de complicaciones.

La identificación de esta anormalidad es posible mediante métodos de imagen, entre ellos la ecografía y la resonancia magnética. Los hallazgos ecográficos que se pueden evidenciar son desaparición o irregularidad de área hipoecoica retro-placentaria, grosor miometrial retro-placentario inferior a $1 \mathrm{~mm}$, múltiples dilataciones vasculares, lagunas placentarias (aspecto de queso suizo) y áreas hipervascularizadas en el Doppler color, que se caracterizan porque el flujo es turbulento con pico de velocidad sistólica > $15 \mathrm{~cm} /$ segundo, presencia de tejido placentario o vasos en la interfase entre la serosa uterina y la vejiga urinaria. La presencia de lagunas placentarias múltiples e irregulares es el signo que aisladamente presenta una sensibilidad elevada (93\%) ${ }^{9}$.

La resonancia magnética, por otro lado, se indica en casos específicos durante el segundo y tercer trimestre de gestación, para descartar compromiso de otro órgano, como vejiga ${ }^{10}$, pero en esta investigación ninguna de las pacientes requirió de este estudio.

Asimismo, los hallazgos intraoperatorios de acretismo más comunes son la distorsión, deformación o levantamiento del segmento uterino inferior (aspecto de medusa), tejido placentario invadiendo la serosa vesical y uterina, e hipervascularización masiva del segmento inferior, como se aprecia en la figura $3{ }^{11}$.

En cuanto al tratamiento aceptado, se describen la cesárea y la histerectomía obstétrica total en el mismo tiempo quirúrgico; sin embargo, está asociada a sangrados masivos (entre 3 a 5 litros), complicaciones severas (lesión ureteral en $17 \%$ de casos), mayor mortalidad (7 \%), estancias en cuidados intensivos y hospitalización prolongadas ${ }^{12,13}$. Es por ello que el surgimiento de nuevas técnicas de abordaje, como la embolización de arterias placentarias, constituyen alternativas para un manejo más seguro del acretismo placentario,

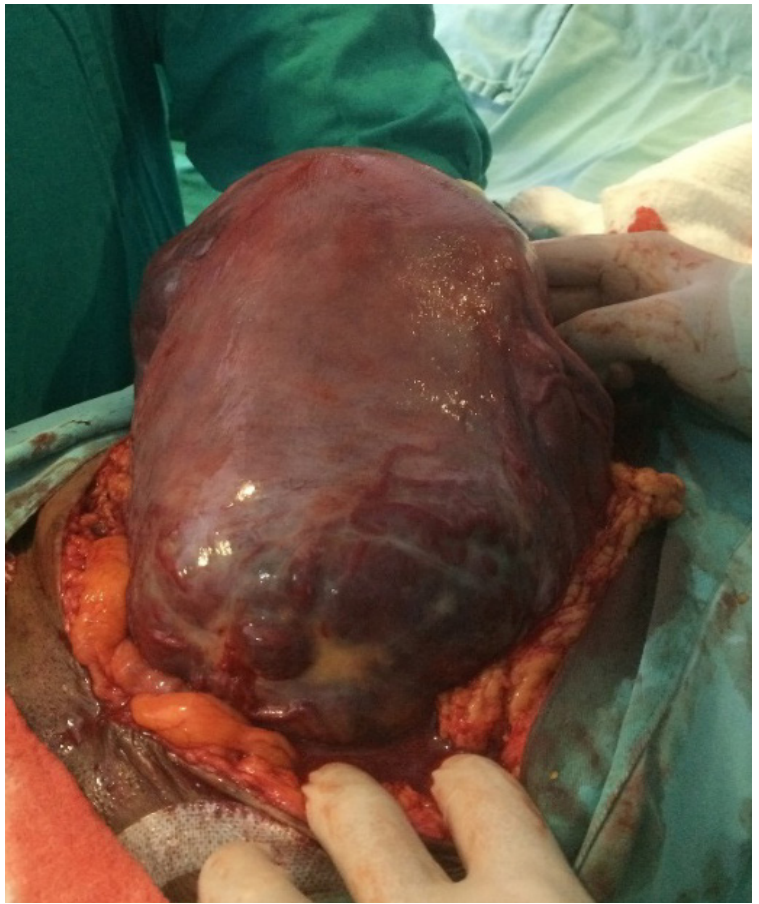

Figura 3. Aspecto macroscópico de los vasos sanguíneos fuera de la serosa uterina, típico de placenta percreta.

que en este protocolo se basó en la disminución del sangrado, permitiendo y estimulando la involución de la placenta con los tiempos de espera y la oclusión endovascular de los vasos anómalos placentarios.

A pesar de que algunos estudios muestran alto riesgo de complicaciones al dejar placenta in situ ${ }^{14,15}$, en esta investigación no se demostró ninguna complicación y se logró que la placenta involucionara adecuadamente. Clausen et al. ${ }^{16}$, en un metaanálisis de casos publicados para el 2014 , encontraron que las mujeres sometidas a embolización sangraban en promedio de 1,25 litros y las que no, entre 2 a 4 litros; además observaron que las pacientes embolizadas requerían transfusión solo en el $28 \%$ de los casos versus el $90 \%$ en las no embolizadas. En este estudio se corroboró que los volúmenes son mucho menores en las pacientes sometidas a embolización, encontrando que el volumen promedio de sangrado fue de $1160 \mathrm{ml}$ y se requirió en promedio la transfusión de 2 unidades de glóbulos rojos por paciente. 
Dentro del manejo médico, se ha utilizado el Metotrexate para disminuir el crecimiento trofoblástico, sin embargo, su uso fue innecesario en estas pacientes, además de que puede aumentar la incidencia de complicaciones (sepsis, sangrados tardíos, inmunosupresión) ${ }^{17}$. Otra opción de manejo es la ligadura de arterias hipogástricas, que fue descrita desde 1960 para el control de diferentes situaciones hemorrágicas en la pelvis, pero es poco recomendada dada sus complicaciones (claudicación glútea, lesión de vena iliaca, lesión de uréter, ligadura de arteria iliaca externa) y poca efectividad (falla para controlar el sangrado en más del $50 \%$ de casos), además de que impide a los cirujanos vasculares realizar procedimientos endovasculares de control de sangrado ${ }^{18,19}$.

El uso de técnicas mínimamente invasivas endovasculares ha revolucionado el tratamiento de muchas enfermedades, logrando controlar sangrados que antes eran imposibles de tratar. Específicamente para el acretismo placentario, se ha usado la canalización de las arterias aorta, iliacas comunes e hipogástricas, con balones que, al ser inflados, ocluyen el flujo sanguíneo, pero se han descrito malos resultados y complicaciones graves (embolismo, disección y ruptura iliaca) ${ }^{19}$.

Es por ello que la embolización uterina como control de sangrado ginecológico se describió por primera vez en 1979 por Brown y Heaston, con efectividad de más del $90 \%{ }^{20}$, y el uso de embolizaciones selectivas para acretismo placentario fue reportado por primera vez en 1992 por el grupo de Bakri y Linjawi ${ }^{21}$, demostrando ser la clave para el control del sangrado, llegando a vasos de menos de 5 milímetros de diámetro, ocluyéndolos de manera ultra-selectiva, sin sacrificar vasos normales o tejidos adyacentes sanos.

La imagen angiográfica debe hacerse con sustracción digital en fase arterial temprana y tardía, para poder observar las ramas arteriales anómalas en sacacorchos pseudotumorales ${ }^{22}$ (figura 4).

En los casos de acretismo placentario, no solamente la embolización de las ramas uterinas controla el sangrado. Es muy importante evaluar la participación de otras arterias como pudendas, epigástricas, ováricas, sacras y obturatriz ${ }^{23}$. Clásicamente, el principal material de embolización utilizado ha sido el Gelfoam (gelatina de piel porcina purificada), pero ocurre reabsorción del material en 4 a 6 semanas, por lo que el uso de agentes más efectivos como las micropartículas y microesferas de polivinilo alcohol (500 a 900 micras), asociada a coils de platino o líquidos embolizantes (como el onyx o glue), permiten una oclusión permanente y segura del vaso elegido ${ }^{24}$ (figura 5).

El éxito en general de la embolización, que se refiere a la oclusión completa de los vasos elegidos (figura 6), oscila entre el 85 y $95 \%{ }^{10}$, y en este trabajo fue del $100 \%$. La embolización ha demostrado en diferentes estudios disminuir estancias hospitalarias, incluidas las de cuidado intensivo, necesitando únicamente en un $25 \%$ de casos este recurso ${ }^{25}$.

La incidencia de complicaciones secundarias a la embolización, oscila entre el 6 y el $9 \%{ }^{26}$, asociadas al acceso vascular femoral (fistulas, pseudoaneurismas, hematomas postpunción) o a la embolización de sitios no indicados con necrosis de estructuras vecinas. Esta última se puede presentar en pacientes con antecedentes

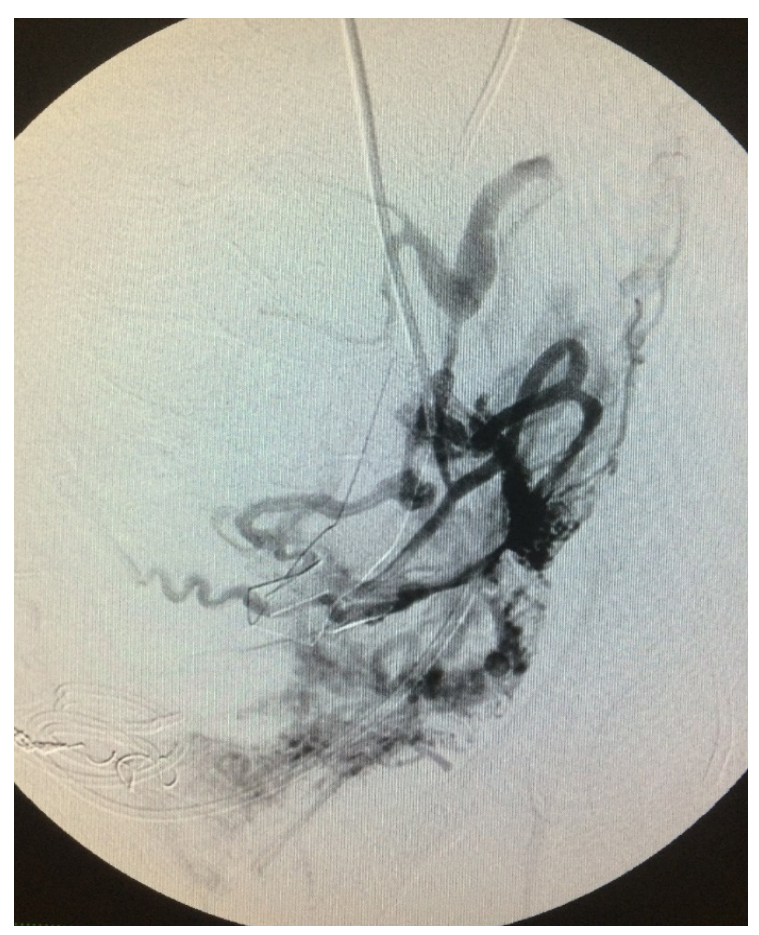

Figura 4. Vasos pseudotumorales anomalos y multiples que nutren la placenta percreta. 


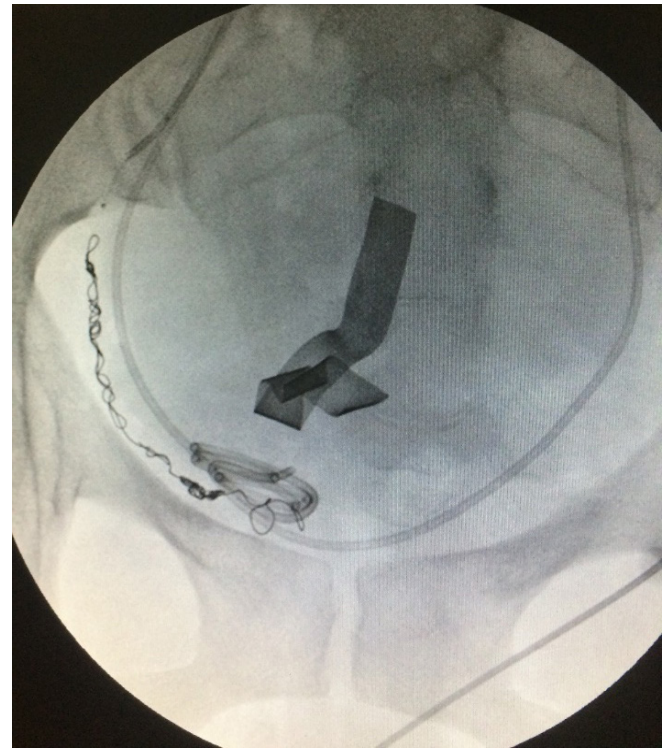

Figura 5. Embolización con microcoils

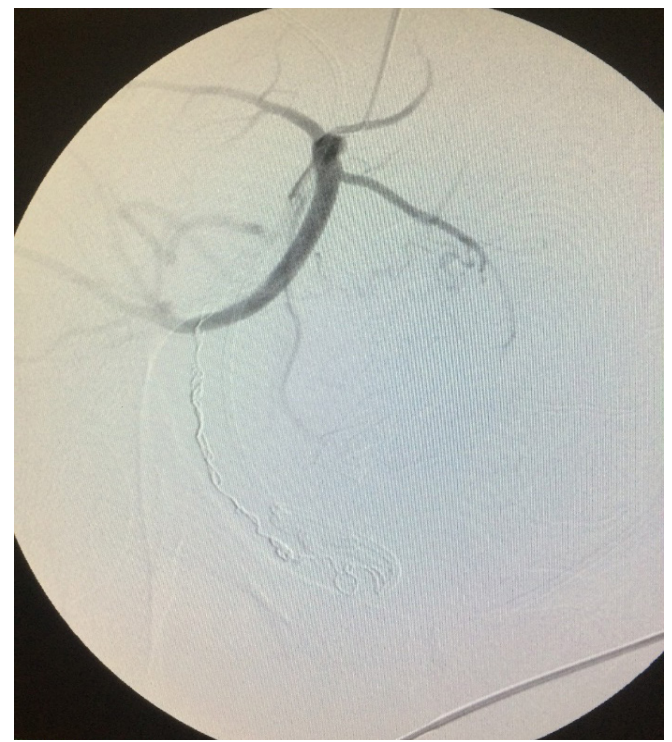

Figura 6. Resultado exitoso con oclusión completa de las ramas elegidas, preservando las arterias glútea y vesical.

de radioterapia abdominal, neoplasias pélvicas o enfermedad oclusiva arterial severa. Otras complicaciones son el síndrome postembolización, abscesos, isquemia de miembros inferiores, o claudicación glútea ${ }^{27}$. En este trabajo no se tuvo ninguna complicación asociada al procedimiento endovascular.
Considerando esta patología como problema de salud pública, debido a su notable aumento en las últimas décadas y su mortalidad, es fundamental tener un protocolo de manejo institucional. Asimismo, es necesario realizar un adecuado control prenatal para la prevención y el diagnóstico temprano del acretismo placentario y, cuando se confirme, direccionar a las pacientes a centros con experiencia en el manejo endovascular, disponibilidad de banco de sangre, cuidado intensivo y demás recursos para el manejo de esta desafiante enfermedad.

\section{Consideraciones éticas}

El presente estudio se enmarca en los principios señalados en la Declaración de Helsinki de la Asociación Médica Mundial, Informe Belmont, la normativa colombiana establecida por la Resolución 8430 de 1993, por la que se establecen las normas científicas, técnicas y administrativas para la investigación en salud. El estudio es catalogado como investigación con riesgo mayor que el mínimo debido a que incluye procedimientos quirúrgicos y endovasculares.

Consentimiento informado. Se obtuvo el consentimiento informado de las pacientes, para el uso de datos de su historia clínica y material fotográfico, respetando la confidencialidad de todos los datos obtenidos en la historia clínica.

Fuentes de financiación. El estudio no conto con ningún tipo de financiación provenientes de agencias del sector público, sector comercial o entidades sin ánimo de lucro.

Conflictos de intereses. Los autores de la investigación declaran no tener ningún conflicto de intereses.

\section{Contribución de los autores}

- Concepción y diseño del estudio: Cesar Eduardo Jiménez-Jiménez, Angy Lorena Meneses-Parra.

- Adquisición de datos: Cesar Eduardo Jiménez-Jiménez, Angy Lorena Meneses-Parra.

- Análisis e interpretación de datos: Cesar Eduardo Jiménez-Jiménez, Angy Lorena Meneses-Parra.

- Redacción del manuscrito: Cesar Eduardo JiménezJiménez, Jorge Ernesto Niño-González, Angy Lorena Meneses-Parra.

- Revisión crítica: Cesar Eduardo Jiménez-Jiménez, Jorge Ernesto Niño-González, Angy Lorena Meneses-Parra. 


\section{Referencias}

1. Yu PC, Ou HY, Tsang LLC, Kung FT, Hsu TY, Cheng YF. Prophylactic intraoperative uterine artery embolization to control hemorrhage in abnormal placentation during late gestation. Fertil Steril. 2009;91:1951-5. https://doi.org/10.1016/j.fertnstert.2008.02.170

2. Tong SYP, Tay KH, Kwek YC. Conservative management of placenta accreta: review of three cases. Singapore Med J. 2008;49:156-9.

3. Silver RM, Barbour KD. Placenta accreta spectrum: accreta, increta, and percreta. Obstet Gynecol Clin North Am. 2015;42:381-402. https://doi.org/10.1016/j.ogc.2015.01.014

4. Véliz F, Núñez A, Selman A. Acretismo placentario: Un diagnóstico emergente. Abordaje quirúrgico no conservador. Rev Chil Obstet Ginecol. 2018;83:51326. https://doi.org/10.4067/s0717-752620180 00500513

5. Vera E, Lattus O, Bermúdez H, Espinoza L, Ibáñez C, Herrera A, Almuna R. Placenta percreta con invasión vesical: reporte de 2 casos. Rev Chil Obstet Ginecol. 2005;70:404-10. https://doi.org/10.4067/S0717-75 262005000600010

6. Tseng SH, Lin CH, Hwang JI, Chen WC, Ho ESC, Chou MM. Experience with conservative strategy of uterine artery embolization in the treatment of placenta percreta in the first trimester of pregnancy. Taiwan J Obstet Gynecol. 2006;45:150-4. https://doi.org/10.1016/S1028-4559(09)60214-X

7. Hernández MLD, Herrera VJE, Mendoza HF, Adaya LEA. Acretismo placentario: Experiencia en obstetricia crítica. Rev Med UV. 2018;18:75-87.

8. Carnevale FC, Kondo MM, de Oliveira-Sousa W, Barbosa-Santos A, da Motta-Leal JM, Mota-Moreira A, et al. Perioperative temporary occlusion of the internal iliac arteries as prophylaxis in cesarean section at risk of hemorrhage in placenta accreta. Cardiovasc Intervent Radiol. 2011;34:758-64.

https://doi.org/10.1007/s00270-011-0166-2

9. Collins SL, Ashcroft A, Braun T, Calda P, Langhoff-Roos J, Morel 0 , et al. Proposal for standardized ultrasound descriptors of abnormally invasive placenta (AIP). Ultrasound Obstet Gynecol. 2016;47:271-5. https://doi.org/10.1002/uog.14952

10. Lim HJ, Kim JY, Kim YD, Park JY, Hong JS. Intraoperative uterine artery embolization without fetal radiation exposure in patients with placenta previa totalis: Two case reports. Obstet Gynecol Sci. 2013;56:45-9. https://doi.org/10.5468/OGS.2013.56.1.45

11. Takahashi H, Hayashi S, Matsuoka K, Kitagawa M. Placenta accreta following uterine artery embolization. Taiwan J Obstet Gynecol. 2010;49:197-8. https://doi.org/10.1016/S1028-4559(10)60041-1
12. Villagómez-Mendoza EA, Yescas-Osorio IN, Toriz-Prado A. Placenta percreta con invasión a vejiga; reporte de un caso y descripción de la técnica quirúrgica. Prog Obstet Ginecol 2019;62:373-8.

13. Tórrez-Morales F, Briones-Garduño JC. Percretismo placentario con invasión de vejiga y recto. Cir Cir. 2017;85:66-9.

https://doi.org/10.1016/j.circir.2015.08.011

14. Takeda A, Koyama K, Imoto S, Mori M, Nakano T, Nakamura H. Conservative management of placenta increta after first trimester abortion by transcatheter arterial chemoembolization: a case report and review of the literature. Arch Gynecol Obstet. 2010;281:381-6. https://doi.org/10.1007/s00404-009-1238-2

15. Höpker M, Fleckenstein G, Heyl W, Sattler B, Emmons G. Placenta percreta in 10 weeks of gestation with consecutive hysterectomy. Human Reprodution. 2002;17:817-20. https://doi.org/10.1093/humrep/17.3.817

16. Clausen C, Lönn L, Langhoff-Roos J. Management of placenta percreta: a review of published cases. Acta Obstet Gynecol Scand. 2014;93:138-43. https://doi.org/10.1111/aogs.12295

17. Sherer DM, Gorelick C, Zigalo A, Sclafani S, Zinn HL, Abulafia O. Placenta previa percreta managed conservatively with methotrexate and multiple bilateral uterine artery embolizations. Ultrasound Obstet Gynecol. 2007;30:227-8. https://doi.org/10.1002/uog.4075

18. Morel O, Malartic C, Muhlstein J, Gayat E, Judlin P, Soyer P, Barranger E. Pelvic arterial ligations for severe post-partum hemorrhage. Indications and techniques. J Visc Surg. 2011;148:e95-e102. https://doi.org/10.1016/j.jviscsurg.2011.02.002

19. Fitzpatrick KE, Sellers S, Spark P, Kurinczuk JJ, Brocklehurst P, Knight M. The management and outcomes of placenta accreta, increta, and percreta in the UK: a population-based descriptive study. BJOG. 2014;121:62-71. https://doi.org/10.1111/1471-0528.12405

20. Josephs SC. Obstetric and gynecologic emergencies: a review of indications and interventional techniques. Semin Intervent Radiol. 2008;25:337-46. https://doi.org/10.1055/s-0028-1102992

21. Bakri YN, Linjawi T. Angiographic embolization for control of pelvic genital tract hemorrhage. Report of 14 cases. Acta Obstet Gynecol Scand. 1992;71:17-21. https://doi.org/10.3109/00016349209007941

22. Campos-Bolaños GA, Enriquez-García R, Guerrero-Avendaño G. Ventajas de la embolización en pacientes con placenta acreta. Anales de Radiología México. 2013;12:236-40.

23. Soyer P, Barat M, Loffroy R, Barral M, Dautry R, Vidal V, et al. The role of interventional radiology in the management of abnormally invasive placenta: a systematic 
review of current evidences. Quant Imaging Med Surg. 2020;10:1370-91.

https://doi.org/10.21037/qims-20-548

24. Lindquist JD, Vogelzang RL. Pelvic artery embolization for treatment of postpartum hemorrhage. Semin Intervent Radiol. 2018;35:41-7.

https://doi.org/10.1055/s-0038-1636520

25. Banovac F, Lin R, Shah D, White A, Pelage JP, Spies J. Angiographic and interventional options in obstetric and gynecologic emergencies. Obstet Gynecol Clin North Am. 2007;34:599-616.

https://doi.org/10.1016/j.ogc.2007.06.004
26. Ojala K, Perälä J, Kariniemi J, Ranta P, Raudaskoski T, Tekay A. Arterial embolization and prophylactic catheterization for the treatment for severe obstetric hemorrhage. Acta Obstet Gynecol Scand. 2005;84:1075-80. https://doi.org/10.1111/j.0001-6349.2005.00727.x

27. Chen C, Lee SM, Kim JW, Shin JH. Recent update of embolization of postpartum hemorrhage. Korean J Radiol. 2018;19:585-96.

https://doi.org/10.3348/kjr.2018.19.4.585 